\title{
Modeling of signaling networks
}

\author{
Susana R. Neves and Ravi lyengar*
}

\begin{abstract}
Summary
Biochemical networks, including those containing signaling pathways, display a wide range of regulatory properties. These include the ability to propagate information across different time scales and to function as switches and oscillators. The mechanisms underlying these complex behaviors involve many interacting components and cannot be understood by experiments alone. The development of computational models and the integration of these models with experiments provide valuable insight into these complex systems-level behaviors. Here we review current approaches to the development of computational models of biochemical networks and describe the insights gained from models that integrate experimental data, using three examples that deal with ultrasensitivity, flexible bistability and oscillatory behavior. These types of complex behavior from relatively simple networks highlight the necessity of using theoretical approaches in understanding higher order biological functions. BioEssays 24:11101117, 2002. ๑ 2002 Wiley Periodicals, Inc.
\end{abstract}

\section{Introduction}

An intracellular signaling cascade can no longer be viewed as a linear pathway that relays and amplifies information. There is now evidence that shows that the cell uses these pathways as a way of integrating multiple inputs to shape a uniquely defined output. ${ }^{(1,2)}$ Hence the interactions of different pathways and the dynamic modulation of the activities of the components within signaling pathways can create a multitude of biological outputs. The cell appears to use these complex networks of interacting pathways and regulatory feedback mechanisms to co-coordinately regulate multiple functions. ${ }^{(3,4)}$ These outputs allow the cell to respond to and adapt to an ever-changing environment. Due to this increasing complexity, it is often not possible to understand intuitively the "systems behavior" of signaling networks. Rather it has become necessary to devel-

Department of Pharmacology and Biological Chemistry, Mount Sinai School of Medicine, New York.

Funding agency: Computational Modeling in the lyengar laboratory is supported by NIH grants GM54508 and CA 81050 . Susan Neves is supported by an individual predoctoral NRSA, F31- GM 65065 from the NIH.

*Correspondence to: Ravi lyengar, Department of Pharmacology and Biological Chemistry, Box 1215, Mount Sinai School of Medicine, One Gustave Levy Place, New York NY 10029.

E-mail: ravi.iyengar@mssm.edu

DOI 10.1002/bies.1154

Published online in Wiley InterScience (www.interscience.wiley.com). op formal models of these sets of interactions and analyze the behavior of these models both to develop a systemslevel understanding and to obtain experimentally testable predictions.

Theoretical analysis of biochemical networks has a long history, and has been successfully applied to the analysis of metabolic pathways and physiological processes. ${ }^{(5,6)}$ Computational modeling has emerged as a powerful descriptive and predictive tool that allows the study of complex systems. ${ }^{(7-9)}$ This approach is becoming increasingly useful in many areas of biology, including in the study of signaling pathways given the identification of a growing number interactions within and between signaling pathways in the cell. The explicit modeling approach should allow the monitoring of the effects of multiple signal inputs that may arrive simultaneously and/or sequentially and the subsequent processing and integration of these signals. Such analysis would lead to understanding of the complexity underlying the higher order functions of signaling networks, and may even help identify novel properties that would not be observable by the study of isolated signaling pathways.

Computational models should represent the biological system as accurately as possible and be able to mimic the behavior of the system over a wide variety of conditions. For this to occur, the model should be based on and fully constrained by experimental data. The amount and quality of the available experimental data will largely determine the fidelity of the model. There is a large and substantial body of literature that provides information on biochemical parameters. Collecting parameter information from the published literature is one important aspect in the development of computational models. However, often this is not sufficient. As these models become more complex, it will become necessary to gather experimental data in an explicit manner for computational analysis. Such data should include cellular concentrations of the components as well as the kinetic constants for interactions between components. Even when most of the required experimental data is available, the development of accurate models requires consideration of a number of issues in terms of the appropriate underlying mathematical assumptions and computing environments. Here we review current approaches to modeling signaling pathways and networks.

\section{Signaling modules and connections maps}

Building complex models of signaling networks is best accomplished in a step-wise manner. One of the first steps involves the definition of reactions involved in terms of 
modules that can be both experimentally constrained and computationally analyzed. In order to do this, operational boundaries, which do not exist in the cell, may have to be created. For example, when modeling growth factor activation of MAP-kinases through receptor tyrosine kinases and the small GTP-binding protein Ras, in order to model the activation of MAP kinase, one may decide to use as an inputactivated Ras. The output may be the dually phosphorylated MAPK or a component further downstream such as the activated transcription factor Elk. Once a valid model has been created for Ras activation of MAPK, one could add another level of activation by modeling the dynamics of the growthfactor-ligand-dependent activation of receptor tyrosine kinase, and the recruitment of adaptor proteins that lead to the activation of Ras. Hence the cascade of protein kinases from Raf1 to MAP kinase pathway could be considered one module, and the growth factor interaction with the receptor tyrosine kinase to activation of Ras could be considered another module. ${ }^{(10)}$ If we want to study how the cAMP pathway inhibits signaling of the growth factor pathway, this can be set up as a third module with the connection between the CAMP and MAP-kinase pathways represented as protein kinase $A$ inhibition of $c$ Raf. ${ }^{(11-13)}$ Thus one can construct a three-module system to computationally study how the activation of Gs-coupled receptors can modulate signal flow from receptor tyrosine kinases to MAP-kinases 1,2. A schematic representation of these modules is shown in Fig. 1. It should be noted that these modules do not correspond to more conventional cell biological boundaries such as membrane-delimited or cytoplasmic events. The boundaries of the modules are often defined by functional input-output relationships. Modules may also reflect spatial locations in the cytoplasm, as defined by protein scaffolds and anchors. For instance, in some systems, Raf, MEK and MAP-kinase are bound to a scaffold. ${ }^{(14)}$

We have found such an approach to be very useful in developing models of signaling pathways that interact with each other to form networks. ${ }^{(1,15)}$ Indeed it has been suggested that, in a general sense, all biological studies may be moving from a molecular to modular phase. ${ }^{(16)}$ The ability to define "modules" has considerable operational advantages during the building of models. It enables the size of individual units to be constrained by including a limited (and manageable) number of components. Additionally, by carefully defining the inputs and outputs of the modules, it is often possible to use experimentally obtained input-output relationships to constrain the overall behavior of the modules. The assembly of the modeled modules in a combinatorial manner can then be used to create complex pathways and networks, and these in turn can be analyzed for the identification of novel emergent properties that the individual modules by themselves do not show. Typically these modules can be written as connection maps, such as those that we have generated for the heterotrimeric G protein pathways at Science's STKE web site. ${ }^{(17)}$ These connections maps are qualitative in nature. The identity of the components and their interactions are defined but quantitative information about both the components and interactions are needed to develop predictive models. There are several databases available that contain information on protein interactions that can be helpful in setting up the initial connections maps. These are listed in Table 1.

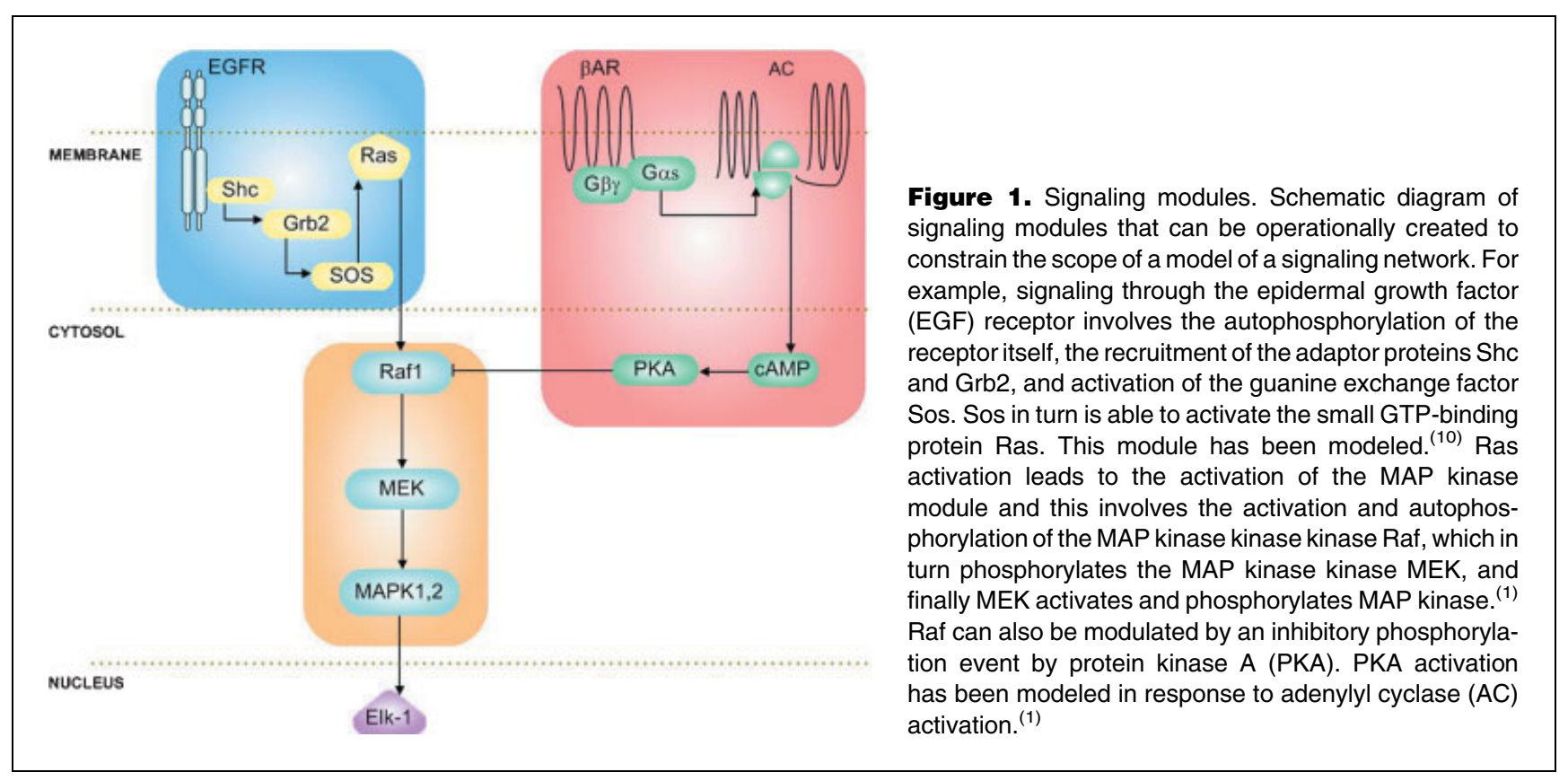


Table 1. Brief list of web-accessible databases containing information on protein-protein interactions and signaling pathways
Database

Alliance for Cell Signaling

Biomolecular Interaction Network Database

Database of Interacting Proteins

Database of Quantitative Cellular Signaling

Kyoto Encyclopedia of Genes and Genomes

PathDB

Proteome

Science's Signal Transduction

Knowledge Environment

Transpath

\section{URL}

www.cellularsignaling.org www.binddb.org

dip.doe-mbi.ucla.edu doqcs.ncbs.res.in

www.genome.ad.jp/kegg/

www.ncgr.org/pathdb/

www.proteome.com

stke.sciencemag.org

transpath.gbf.de/

\section{Reaction parameters}

Once the connections maps have been set up, the next step is to collect the parameter information needed for each of the components and their interactions (Fig. 2). This involves concentrations for each component, kinetic rate constants for interactions and enzymatic reactions, and diffusion rates, if the model involves spatial parameters. Some of this information is available in the original biochemical literature. However, in most cases, the quantitative information has to be manually extracted from the primary literature and entered into the database. This is a laborious process and is possible only for components that have been purified from native sources.
An initial version of such database has now been created by Bhalla and co-workers (DOQCS, Database of Quantitative Cellular Signaling URL: docqcs.ncbs.res.in) and has been used in our quantitative models. Although the information in this database is limited, it is detailed and annotated. Indeed with data extraction from the primary literature, annotation and explicit statement of assumptions becomes a critical factor in defining the biochemical parameters. Contextual information is crucial for parameter selection and development of realistic models. These criteria are likely to be increasingly important as large-scale data-gathering efforts get underway. The quantitative data in these endeavors need to be appropriately annotated so that the larger biological community can use them.

A reasonably clear division currently exists for components and interactions for which quantitative parameters can be estimated versus those for which it is currently not feasible. Proteins that were biochemically purified prior to cDNA cloning most often have a reasonable amount of biochemical data available. However, with the advent of homology cloning and yeast two-hybrid screening techniques in molecular biology, many proteins were first known from their cDNA clones. Here the biochemical data is obtained from heterologous expression systems such as bacteria or insect cells and it is not entirely clear how representative the reaction parameters obtained from the heterologous systems are of the native proteins. Additionally almost always missing is the cellular concentrations of key components in the native systems.

If parameter information is scarce or incomplete, it is sometimes feasible to create models that will fit the observed input-output relationship data. ${ }^{(18)}$ This approach can yield valuable models, especially if there are a lot of experimental data available that can be used to constrain the model. In cases

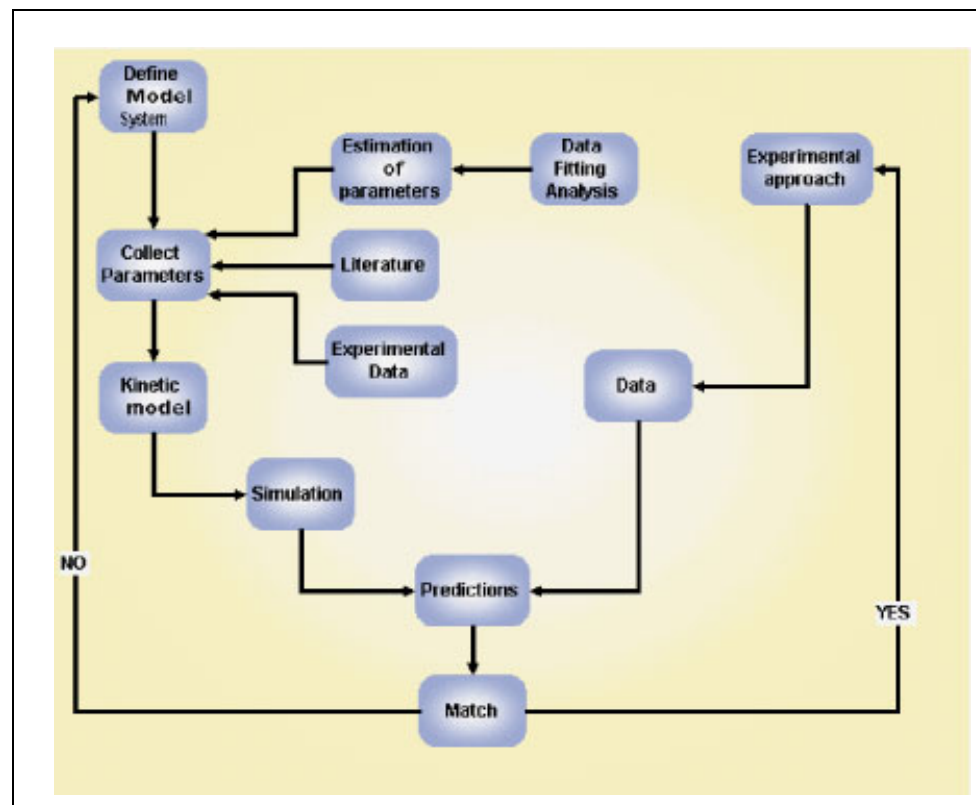

Figure 2. Flow diagram of the steps involved in the creation of a computational model. Initially, the model is defined as a connection map based on available biological data. Once the reaction schemes are set up, parameter information is collected from the existing biochemical literature; input-output experimental data that constrain the model are also collected. Parameters may be estimated and obtained with the use of data-fitting analysis software. Once all the parameters are obtained, a kinetic model is developed and a simulation is created. The simulation will give rise to predictions that can be compared with the experimental data.

\section{BioEssays 24.12}


where the mechanistic details are unclear, it is sometime possible to fill in the gaps with the simplest mechanisms available. The assumption can then be subsequently experimentally verified. Another option would be to use mechanisms implemented by similar pathways. However all of these assumptions need to be carefully evaluated and explicitly considered. Almost all models have some assumptions and it is imperative that the costs of the assumptions in creating the models be weighed against the potential benefits in terms of predictions and systems-level understanding. Predictions are especially valuable since they can lead to iterative experimental constraining of the models. By use of these approaches, one can set detailed models of kinetic reaction schemes. Many examples that have been used by Bhalla and lyengar are available at DOQCS (URL: doqcs.ncbs.res.in). A summary of the steps involved in the modeling is shown schematically in Fig. 2.

\section{Simulations}

The completed reaction schemes can then be used to create simulations, and these simulations can be compared with the experimentally observed input-output relationships. On the one hand, if the simulations can mimic with reasonable accuracy the characteristics observed in the original experimental system then the overall mechanism predicted in the model is likely to be valid. A valid model can then be analyzed to obtain predictions about any novel properties and higher order cellular capabilities that the signaling network may possess. This can be done by parameters variation and altering the internal connectivities to represent various biological states, and determining how these changes may modulate the output(s) of the network. Such manipulations can give rise to new experimental questions, and be useful in developing a better understanding of the organizational principles that the cell uses to regulate information flow.

If, on the other hand, the simulations fail to mimic the experimental results, the mechanism proposed is faulty or incomplete and more experimental data is needed to better understand the biological system and define the model. In this case, the model can still be informative since it may help identify the types of data that it are still unknown and are needed for a better understanding of the system. Thus both successes and failures in model development can be of value in defining new experiments.

\section{Modeling methods}

Several modeling environments are now available that can be used to develop kinetic simulations of signaling pathways and networks. These programs can simulate the steady-state and kinetic behavior of reactions. There are at least ten programs currently available and space limitations preclude a detailed discussion of each. A listing of these programs and links are available at the DOQCS site. Each of these software suites has features that make them particularly suitable for specific purposes. Due to limitations in our expertise, we have selected three programs that we use in our laboratory for further description. At their core, all of the modeling programs use built-in differential equations and solve them, in a transparent manner. Generally, the user does not need to specify the mathematical methods required for the simulation or be knowledgeable about the programming codes used. This allows the user with limited mathematical/computational background to develop and analyze models. Although detailed technical knowledge is not necessary, it is essential that the appropriate mathematical representation for the biological system that is being studied is used. For this, the practicing biologist will need to have an adequate understanding of mathematics. The results from the simulation can then be plotted using built-in plotting programs or the data can be easily exported to spreadsheets. In addition to the specialized programs for biological simulations, general mathematical analysis programming environments such as MATLAB or MATHEMATICA can also be used for biological models.

The three programs that we use are briefly described below. Each program has its own key features and all three of them can be used in a coordinated fashion to complement each other and optimize different aspects of a single model.

\section{Genesis/KinetiKit:}

(URL: www.ncbs.res.in/ bhalla/kkit/index.html)

Kinetikit is a graphical simulator for modeling signaling networks ${ }^{(1)}$. It runs with the large-scale General Neural Simulation System (GENESIS). This modeling program has been widely used for the simulation of biological pathways in our laboratory. It has a user-friendly graphical interphase and substantial computational power. Since it uses a UNIX platform both KinetiKit and Genesis are very stable and fast. Although this is a very useful program, it has limitations as currently configured. Genesis can solve only ordinary differential equations (ODE) and hence the program can only use a deterministic approach to modeling that assumes a "well-stirred" reaction where every molecule has equal access to any other molecule. Often such deterministic models are quite valid and in such cases the KinetiKit-GENESIS platform is quite useful.

\section{Gepasi 3 (URL: www.gepasi.org)}

GEPASI 3 is a modeling platform that allows the simulation of biochemical pathways. ${ }^{(19)}$ GEPASI 3 runs on the Windows operating system and is able to carry out steady-state and time-course simulations. GEPASI provides the user with several choices of output for the data generated in the simulation, in a graphical form or in a spreadsheet form. One feature of GEPASI that is useful is its parameter scan capability. ${ }^{(20)}$ The user is able to select the parameter that will be varied, and the range and extent of the variation and 
develop a set of simulations that can be compared with experimentally observed data. This feature can then be used to optimize, fit or even estimate unknown parameters to simulate experimentally observed input-output relationships.

\section{Virtual Cell (URL: www.nrcam.uchc.edu)}

Virtual Cell is a computational environment that uses a webbased graphical interface for the user to input the data needed for the model. ${ }^{(21)}$ This usually involves a collection of molecules, with their respective parameters and subcellular localizations. Virtual Cell is especially useful when the model requires a strict spatial organization, which allows a more geometrical model where the flux between compartments can be simulated. The model is built using a graphical interface that allows the user to determine the geometry of the model. Virtual Cell uses two separate numerical solvers: one for their kinetic modeling that uses Ordinary Differential Equations (ODEs) and another for their spatial modeling that uses Partial Differential Equations (PDEs). The output of data in Virtual Cell is in different formats: spreadsheets, pseudocolor images that can represent the modulation of spatially restricted molecules over time, and QuickTime movies.

\section{Emerging concepts from models of signaling and biochemical networks}

There are several emerging concepts that are likely to be key elements of the organizational principles used by the cell. These concepts are being discovered with the use of computational modeling and experimentation that are closely linked to the models. Some of these concepts include ultrasensitivity, bistability and rhythmic behavior. This is undoubtedly a partial list and one of the future goals will be to develop a more complete list of systems-level properties of a cell. However, it is nevertheless useful to consider how these systems-level properties are being currently identified and the underlying molecular mechanisms elucidated. We consider three examples where feedback loops, which are a ubiquitious feature of biological systems, can be adapted to yield distinct systems-level properties.

\section{Ultrasensitivity}

Ultrasensitivity, a term used in quantitative biochemistry defines a response that is more sensitive to ligand concentration as compared to standard responses defined by the MichaelisMenten equation. ${ }^{(22,23)}$ When applied to signaling pathways ultrasensitivity indicates that a response is more sensitive to extracellular stimulation than the normal hyperbolic response. A classical Michaelis-Menten reaction exhibits a simple hyperbolic reaction velocity curve, while an ultrasensitive reaction displays a sigmoidal curve. One of best examples of ultrasensitive responses has come from the work of Ferrell and Machlesder $^{(24)}$ on Xenopus oocytes, where they are able to show ultrasensitive responses in the MAP-kinase pathway to progesterone.

Progesterone triggers the maturation of oocytes by promoting their exit from a $G_{2}$ phase arrest and their further progression into meiosis, where they are arrested at metaphase of meiosis II until fertilization. This re-entry into the cell cycle is dependent on the activation of the MAP-kinase cascade by progesterone and the eventual formation of the cyclindependent kinase 2 and cyclin B complex. ${ }^{(25-27)}$ This phenomenon is digital (the oocytes mature or they fail to mature) and irreversible (once they mature they remain in their mature form). Ferrell and co-workers had initially shown that oocyte cell extracts with activated Mos (the MAP-kinase kinase Kinase in this system) display an ultrasensitive MAPK response. ${ }^{(28)}$ Thus activation of Mos leads to the creation of one of two species of MAPK, the phosphorylated or the nonphosphorylated. Rarely are both species seen simultaneously, which suggests a highly cooperative cascade. MAP-kinase activation by Mos displays a Hill coefficient of 5 suggesting extensive cooperativity and ultrasensitivity.

Interestingly, when a population of oocytes was treated with progesterone, the overall response resulted in graded MAPK activity. ${ }^{(24)}$ This response showed Michaelis-Menten kinetics and a Hill coefficient of 1 (no cooperativity or ultrasensitivity). However, when the MAPK response of individual oocytes to progesterone was quantitated, a clear all or none response is observed. Thus each individual oocyte responded to a specific concentration of progesterone with either complete activation of MAPK or no activation of MAPK. This behavior can best be described as a switch-like response. The observed ultrasensitivity response has been linked to the intrinsic ultrasensitivity in the requirement of the MAPK cascade on its non-processive dual phosphorylation mechanism for activation. There is also the contribution of a positive feedback loop that appears to increase Mos levels transcriptionally. ${ }^{(24,29)}$

Models were developed where the effects of ultrasensitivity and the feedback loop on the activation of the MAPK were assessed. Together, ultrasensitivity and the feedback loop yield a sharp switch-like response. The model provided valuable insight into determining that both ultrasensitivity (the intrinsic cooperativity of the pathway) and the positive feedback loop were responsible for the observed response. Thus in this case the model was very useful in giving mechanistic insight into the cellular machinery involved in the switch-like behavior.

\section{Flexible bistability}

Within signaling pathways, feedback loops can give rise to switching properties that allow for information propagation across time scales. Bhalla and lyengar (1999) had proposed a positive feedback loop within the growth-factor-stimulated MAP-kinase pathway. MAP-kinase is known to activate cytoplasmic-phospholipaseA2, which produces arachadonic

\section{BioEssays 24.12}


acid, which can activate the typical forms of protein kinase $C$ at basal levels of diacylglycerol. ${ }^{(30-33)}$ The protein kinase $\mathrm{C}$ in turn activates Raf, the most-upstream protein kinase in the MAP-kinase cascade. ${ }^{(34,35)}$ This feedback loop behaves as a switch such that a brief growth factor stimulus can lead to sustained activation of MAP-kinase. The activation of MAP-kinase leads to the induction of MAP-kinase phosphatase, a dual specificity phosphatase that can deactivate MAPkinase. ${ }^{(36)}$ This simple network is shown in Fig. 3A. The coupling of the positive and negative feedback loops could

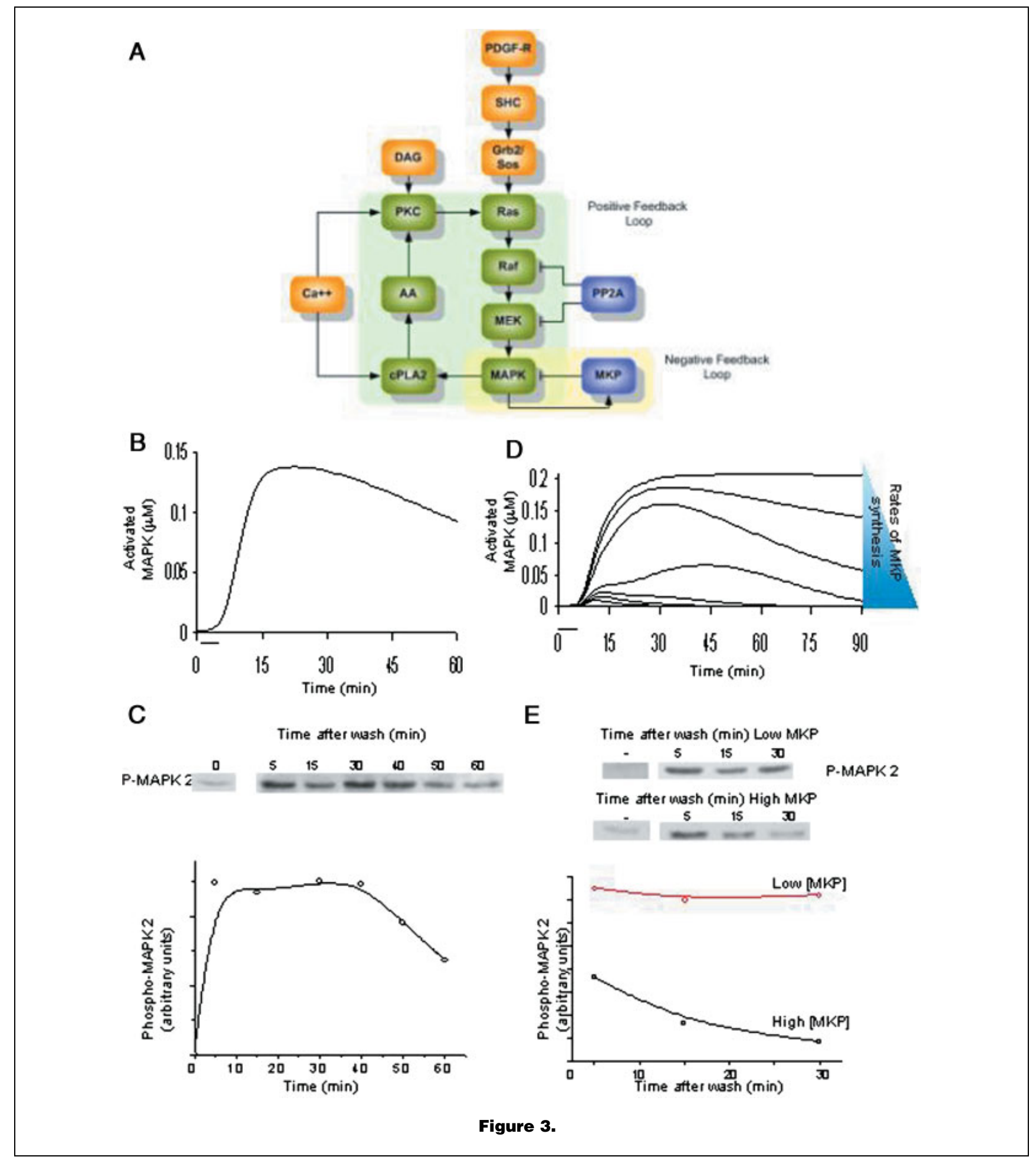

BioEssays 24.12 1115 
under certain conditions lead to oscillatory behavior. However, detailed simulations indicated that such behavior does not appear to occur in a physiologically relevant manner. ${ }^{(37)}$ Further analysis has shown that the induction of MAP-kinase phosphatase can move the system from a bistable to a monostable state. In the monostable state, the system is responsive in a proportional manner to varying concentrations of growth factor ligands. These systems-level predictions were tested in a model system, NIH-3T3 fibroblasts. Here, it was experimentally observed that brief (5 minute) treatment with the growth factor PDGF resulted in sustained (for over 40 minutes) activation of MAP-kinase and that the feedback loop required the activities of both phospholipase A2 and protein kinase $\mathrm{C}$. This is shown in Fig. 3B and C. Fig. 3B shows the simulation and Fig. $3 \mathrm{C}$ the activation of MAP kinase in NIH-3T3 cells after a 5 minute stimulation by PDGF. According to the simulation, when the MAP-kinase phosphatase levels are low the system was capable of responding in a switch-like manner with sustained activation of MAP-kinase after a brief stimulus (Fig. 3D, upper curves). However, upon the induction of MAP-kinase phosphatase, sustained activation of MAP-kinase is not observed. This is shown in the middle and lower curves of Fig. 3D. Thus, at high MAP kinase phosphatase levels, only an early proportional response in MAP kinase activation was observable. ${ }^{(38)}$ This predicted behavior can be experimentally observed in NIH-3T3 cells, at low MKP levels, a 5 minute PDGF stimulation results in MAP kinase being activated for more than 30 minutes. While at high MKP levels, MAP kinase is deactivated between 15 and 30 minutes (Fig. 3E). The MAP kinase pathway can show both monostable and bistable behavior and can move between these two states in a regulated manner. These observations indicate that the system is designed in a flexible manner. Here the systems-level predictions were very useful in designing appropriate experiments. The experiments allowed us to observe systems type behavior that we do not normally look for in molecular analysis. Additionally, one interesting aspect of this study was the identification of MAP-kinase phosphatase as the locus of flexibility. Thus, we are able to ascribe systems-level properties to key components.

\section{Rhythmic behavior}

Two examples described above represent natural systems that are analyzed for systems properties using a combination of experimental and computational approaches. There are also interesting "reverse engineering" approaches being used to create artificial systems that display higher order behavior that is widely observed in natural systems. Several such gene regulatory networks have been recently reviewed. ${ }^{(39)}$ Here we discuss one example. Elowitz and Leibler have recently constructed a gene regulatory network that functions as a synthetic oscillator. ${ }^{(40)}$ This network involves three transcriptional repressors organized in a negative feedback loop and monitored by the expression of the reporter Green Fluorescent Protein (GFP). This type of network created oscillations that could be monitored by observing the GFP expression and the resultant fluorescence in $E$. coli colonies. The oscillatory behavior was simulated using both kinetic and stochastic models that describe the modulation of the components over time. Periodicity in this oscillatory system was in hours and thus the oscillatory cycles were longer than the bacterial cell cycle. The oscillations were present even after the bacterium divided, and they remained correlated in some of the siblings for some time. A noteworthy aspect of this study was the biological system that was developed to fit the design of the model. Thus the biological system was optimized to yield the desired output. The optimization consisted of using strong promoters that could be effectively modulated by the repressor protein, and of decreasing the half-life of the repressor protein by adding a degradation tag. Of course such studies can be criticized as being too artificial and not providing insight into a natural system. While the system is undoubtedly artificial, this

Figure 3. An example of a modeling study comparing modeling predictions and experimental data. A: Schematic representation of the proposed MAP-kinase network. ${ }^{(38)}$ Boxed in light green are the components involved in the positive feedback loop. Boxed in yellow are the components involved in the negative feedback loop. Abbreviations: PDGF-R, platelet-derived growth factor receptor; MEK, MAP or ERK kinase; MAPK, MAP-kinase; MKP, MAP-kinase phosphatase; PP2A, protein phosphatase 2A; cPLA2, cytoplasmic phospholipase A2; AA, arachadonic acid; PKC, protein kinase C; DAG, Diacylglycerol. B: Simulation of MAP kinase activation levels as a function of time after 5 minute PDGF stimulation (bar depicts stimulation period). C: MAP kinase activation in NIH-3T3 cells after 5-minute PDGF stimulation. After stimulation cells were washed and MAP-kinase activity measured at indicated times. Top panel shows phospho-MAP kinase immunoblot. It can be seen a brief stimulation by PDGF leads to sustained activation of MAP-kinase. D: Simulation of MAP kinase activation levels as a function of time with varying concentrations of MAP kinase phosphatase (MKP) as achieved by varying rates of synthesis of MKP, denoted by the blue marker. Lowest rates of MKP synthesis are at the top and highest at the bottom. E: Effect of MKP levels on MAP kinase activation state in NIH3T3 cells. Cells were serum starved and then stimulated with either vehicle (red trace) or PDGF (black trace) for 5 minutes to induce MKP synthesis. The cells were serum starved again for 60 minutes and re-stimulated with PDGF for 5 minutes. MAP-kinase-activity was measured at indicated times after second stimulation Top panel shows phospho-MAP kinase immunoblots corresponding to the restimulated cells (bottom) or the naïve cells (top). It can be seen that induction of MKP leads transient activation of MAP-kinase. Experiments are described in detail in Reference 38. (Figure reprinted from Bhalla US, Ram PT, lyengar R. MAP kinase phosphatase as a locus of flexibility in a nitrogen-activated protein kinase network. Science 2002; 297:1018-1023, with permission from Science Press).

\section{BioEssays 24.12}


reverse-engineering approach provides quite a bit of insight into natural processes. For instance, a large set of behaviors was observed suggesting that even such a well-defined system can be subjected to effects from the internal environment of the bacterial cell. The origins of this complex behavior are not fully understood. However, having a well-defined system may make it easier for the study of the environmental effects. Future analysis will tell if the construction and analysis of artificial systems that display naturally observed behavior will be useful in understanding the mechanisms underlying complex behaviors in biological systems.

\section{Conclusions}

The dramatic progress in biochemistry and molecular biology and the completion of the sequences of many genomes, including that of the human have provided us with a reasonably detailed "parts list" of the cell. From this part lists and our current knowledge of cellular regulatory systems, we have the means to understand the principles underlying the dynamic behavior of cells. This will require an integration of theoretical and experimental approaches at a variety of levels. Here we have described some of the approaches that are currently being applied to study of simple biochemical networks including intracellular networks. Hopefully, the individual principles that can be gleaned from the study of the simple networks can be assembled into an interacting set to yield higher order general principles of dynamical cell function.

\section{References}

1. Bhalla US, lyengar R. Emergent properties of networks of biological signaling pathways. Science 1999;283:381-387.

2. Weng G, Bhalla US, lyengar R. Complexity in biological signaling systems. Science 1999;284:92-96.

3. Jordan JD, lyengar R. Modes of interactions between signaling pathways. Biochem Pharmacol 1998;55:1347-1352.

4. Jordan JD, Landau EM, Iyengar R. Signaling networks: the origins of cellular multitasking. Cell 2000;103:193-200.

5. Heinrich R. Mathematical models of metabolic systems: general principles and control of glycolysis and membrane transport in erythocytes. Biomed Biochim Acta 1985;44:913-927.

6. Leclercq J, Dumont JE. Boolean analysis of cell regulation networks. J Theor Biol 1983;104:507-534

7. Ideker T, Galitski T, Hood L. A new approach to decoding life: systems biology. Annu Rev Genomics Hum Genet 2001;2:343-372.

8. Tyson JJ, Chen K, Novak B. Network dynamics and cell physiology Nat Rev Mol Cell Biol 2001;2:908-916.

9. Firth CA, Bray D. Stochastic Simulation of Cell Signaling Pathways. In: Bower JM, Bolouri H, editors. Computational Modeling of Genetic and Biochemical Networks. Cambridge, Massachusetts: The MIT Press; 2001.

10. Kholodenko BN, Demin OV, Moehren G, Hoek JB. Quantification of short term signaling by the epidermal growth factor receptor. J Biol Chem 1999;274:30169-30181.

11. Wu J, Dent P, Jelinek T, Wolfman A, Weber MJ, Sturgill TW. Inhibition of the EGF-activated MAP kinase signaling pathway by adenosine $3^{\prime}, 5^{\prime}$ monophosphate. Science 1993;262:1065-1069.

12. Mischak H, Seitz T, Janosch P, Eulitz M, Steen H, Schellerer M, Philipp A, Kolch W. Negative regulation of Raf-1 by phosphorylation of serine 621 Mol Cell Biol 1996;16:5409-5418.
13. Hafner S, Adler HS, Mischak H, Janosch P, Heidecker G, Wolfman A, Pippig S, Lohse M, Ueffing M, Kolch W. Mechanism of inhibition of Raf-1 by protein kinase A. Mol Cell Biol 1994;14:6696-6703.

14. Schaeffer HJ, Catling AD, Eblen ST, Collier LS, Krauss A, Weber MJ. MP1: a MEK binding partner that enhances enzymatic activation of the MAP kinase cascade. Science 1998;281:1668-1671.

15. Bhalla US, lyengar R. Functional modules in biological signalling networks. Novartis Found Symp 2001;239:4-13.

16. Hartwell LH, Hopfield JJ, Leibler S, Murray AW. From molecular to modular cell biology. Nature 1999;402:C47-C52.

17. Neves SR, Ram PT, Iyengar R. Heterotrimeric G Proteins pathways. Science 2002;296:1636-1939 stke.sciencemag.org

18. Bhalla US. The Network Within: Signaling Pathways. In: Bower JM, Beeman D, editors. The Book of Genesis. New York: Springer-Verlag; 1998.

19. Mendes P. Biochemistry by numbers: simulation of biochemical pathways with Gepasi 3. Trends Biochem Sci 1997;22:361-363.

20. Mendes P, Kell D. Non-linear optimization of biochemical pathways: applications to metabolic engineering and parameter estimation. Bioinformatics 1998;14:869-883.

21. Loew LM, Schaff JC. The Virtual Cell: a software environment for computational cell biology. Trends Biotechnol 2001;19:401-406.

22. Koshland DE, Jr, Goldbeter A, Stock JB. Amplification and adaptation in regulatory and sensory systems. Science 1982;217:220-225.

23. Goldbeter A, Koshland DE Jr. An amplified sensitivity arising from covalent modification in biological systems. Proc Natl Acad Sci USA 1981;78:6840-6844

24. Ferrell JE, Jr, Machleder EM. The biochemical basis of an all-or-none cell fate switch in Xenopus oocytes. Science 1998;280:895-898.

25. Ferrell JE Jr. Building a cellular switch: more lessons from a good egg. Bioessays 1999;21:866-870

26. Nebreda AR, Ferby I. Regulation of the meiotic cell cycle in oocytes. Curr Opin Cell Biol 2000;12:666-675

27. Ferrell JE, Jr. Xenopus oocyte maturation: new lessons from a good egg. Bioessays 1999;21:833-842.

28. Huang CY, Ferrell JE, Jr. Ultrasensitivity in the mitogen-activated protein kinase cascade. Proc Natl Acad Sci USA 1996;93:10078-10083.

29. Huang CY, Ferrell JE Jr. Dependence of Mos-induced Cdc2 activation on MAP kinase function in a cell-free system. EMBO J 1996;15:2169-2173.

30. Lin LL, Wartmann M, Lin AY, Knopf JL, Seth A, Davis RJ. CPLA2 is phosphorylated and activated by MAP kinase. Cell 1993;72:269-278.

31. Nemenoff RA, Winitz S, Qian NX, Van Putten V, Johnson GL, Heasley LE. Phosphorylation and activation of a high molecular weight form of phospholipase A2 by p42 microtubule-associated protein 2 kinase and protein kinase C. J Biol Chem 1993;268:1960-1964.

32. Nishizuka Y. Intracellular signaling by hydrolysis of phospholipids and activation of protein kinase C. Science 1992;258:607-614.

33. Shinomura T, Asaoka Y, Oka M, Yoshida K, Nishizuka Y. Synergistic action of diacylglycerol and unsaturated fatty acid for protein kinase $C$ activation: its possible implications. Proc Natl Acad Sci USA 1991;88: 5149-5153.

34. Kolch W, Heidecker G, Kochs G, Hummel R, Vahidi H, Mischak H, Finkenzeller G, Marme D, Rapp UR. Protein kinase C alpha activates RAF-1 by direct phosphorylation. Nature 1993;364:249-252.

35. Carroll MP, May WS. Protein kinase C-mediated serine phosphorylation directly activates Raf-1 in murine hematopoietic cells. J Biol Chem 1994; 269:1249-1256.

36. Brondello JM, Brunet A, Pouyssegur J, McKenzie FR. The dual specificity mitogen-activated protein kinase phosphatase- 1 and -2 are induced by the p42/p44MAPK cascade. J Biol Chem 1997;272:1368-1376.

37. Bhalla US, Iyengar R. Robustness of the bistable behavior of a biological signaling feedback loop. Chaos 2001;11:221-226.

38. Bhalla US, Ram PT, lyengar R. Map kinase phosphatase as a locus of flexibility in a nitrogen-activated protein kinase network. Science 2002; 297:1018-1023

39. Ferrell JE. Self-perpetuating states in signal transduction: positive feedback, double-negative feedback and bistability. Curr Opin Cell Biol 2002;14:140-148

40. Elowitz MB, Leibler S. A synthetic oscillatory network of transcriptional regulators. Nature 2000;403:335-338. 